\title{
Permeabilidad a los cloruros del hormigón armado situado en ambiente marino sumergido
}

\section{Chloride permeability of reinforced concrete in submerged marine environment}

\author{
Miguel Ángel Bermúdez O driozola*, Pilar Alaejos Gutiérrez*
}

* Laboratorio Central de Estructuras y Materiales, CEDEX, Madrid, ESPAÑA

mabermud@cedex.es

Fecha de recepción: 10/ 12/2006

Fecha de aceptación: 20/ 02/ 2007

PAG. $15-22$

Resumen

El objetivo del presente trabajo ha sido estudiar el comportamiento del hormigón armado utilizado en los cajones de dos muelles construidos en la costa Mediterránea con hormigón con resistencia a compresión a 28 días de 25 M Pa y contenido de cemento igual a $300 \mathrm{~kg} / \mathrm{m}^{3}$. Los cajones estudiados permanecen sumergidos permanentemente. Las conclusiones preliminares señalan que en el hormigón sumergido la velocidad de difusión de cloruros es mucho más elevada de lo que cabría esperar, pero este efecto queda compensado por la ausencia de oxígeno, la cual eleva notablemente el umbral de cloruros necesario para el inicio de la corrosión.

Palabras Clave: Hormigón armado, corrosión, ambiente marino sumergido, cajones flotantes

Abstract

The purpose of the present study was to analyze the performance of reinforced concrete with 28-day compressive strength of $25 \mathrm{MPa}$ and cement content of $300 \mathrm{~kg} / \mathrm{m}^{3}$ used in caissons sustaining two wharfs on the Mediterranean coast. The caissons studied are permanently submerged. The preliminary conclusions suggest that whereas the rate of chloride penetration in submerged concrete is much higher than expected, the absence of oxygen offsets the deleterious effects of this development by substantially raising the chloride ion concentration required to initiate corrosion.

Keyw ords: Reinforced concrete, corrosion, submerged marine environment, caissons

\section{Introducción}

La mayoría de las aguas de mar presentan una composición química uniforme, caracterizada por la presencia de un $3.5 \%$ de sales solubles en peso. Las mayores concentraciones iónicas son las del $\mathrm{Na}^{+}$y del $\mathrm{Cl}^{-}$, que son de 11.000 y $20.000 \mathrm{mg} / \mathrm{litro}$, respectivamente. También hay cantidades significativas de $\mathrm{Mg}^{2+} \mathrm{y} \mathrm{SO}_{4}{ }^{2-}$, normalmente 1.400 y $2.700 \mathrm{mg} / \mathrm{litro}$, respectivamente. El pH varía entre 7.5 y 8.4 (M ehta y Monteiro, 1986).

Por lo tanto, el hormigón expuesto a un ambiente marino puede deteriorarse debido a los efectos combinados de la acción química de estos constituyentes del agua de mar sobre los productos de hidratación del cemento, de la expansión álcali-árido (cuando hay áridos reactivos), de la presión de cristalización de sales en el hormigón (si una cara de la estructura está sometida a condiciones de humedad y la otra a condiciones de secado), a la acción del hielo en climas fríos, a la corrosión de las armaduras y a la erosión física debida a la acción de las olas y de las partículas en suspensión. Cada una de estas acciones provoca un aumento de la permeabilidad del hormigón, lo que contribuye a que progrese el ataque de la causa inicial y el de los demás tipos de acción (Mehta y Monteiro, 1986). De todas estas posibles patologías, la corrosión de las armaduras suele ser la principal causa de deterioro del hormigón en estructuras de hormigón armado expuestas al agua de mar.

La normativa internacional de aplicación para el proyecto de obras marinas ha ido progresivamente incrementando las exigencias relativas a la calidad del hormigón (en cuanto a tipo y contenido mínimo de cemento y relación a/c máxima), de modo que éste no sufra una degradación significativa durante la vida útil de la estructura.

Así, la actual Instrucción Española de Hormigón 


\section{Procedimiento experimental}

Estructural EHE (M. Fomento, 1998) exige, para el ambiente marino sumergido, utilizar hormigones de relación agua/cemento inferior a 0.50 , contenido de cemento superior a $325 \mathrm{~kg} / \mathrm{m}^{3}$, el empleo de un cemento de características marino-resistentes (M R) y un recubrimiento superior a $45 \mathrm{~mm}$. O tras normativas internacionales tienen requisitos similares o incluso más restrictivos, como la BS 6349 (BSI, 1984) para estructuras marítimas (relación a/c inferior a 0.50 , contenido de cemento superior a 350 $\mathrm{kg} / \mathrm{m}^{3}$ y recubrimiento superior a $50 \mathrm{~mm}$ ), el $\mathrm{ACl} 357 \mathrm{R}$ $(\mathrm{ACl}, 2002)$ para estructuras offshore (relación a/c inferior a 0.45 y recubrimiento superior a $50 \mathrm{~mm}$ ) o las recomendaciones del Comité Técnico 32-RCA de la RILEM (RILEM , 1985) (relación a/c inferior a 0.50, contenido de cemento superior a $350 \mathrm{~kg} / \mathrm{m}^{3}$ y recubrimiento superior a $45 \mathrm{~mm}$ ) y del FIP (FIP, 1985) (relación a/c inferior a 0.45 , contenido de cemento superior a $320 \mathrm{~kg} / \mathrm{m}^{3}$ y recubrimiento superior a $50 \mathrm{~mm}$ ).

Sin embargo, en España durante muchos años el hormigón utilizado en ambiente marino sumergido ha sido de categoría H-25 (25 MPa de resistencia a compresión a los 28 días) ó incluso inferior, incumpliéndose los actuales requisitos del contenido de cemento y relación a/c. En cuanto al contenido cloruros en el hormigón armado, la EHE permite hasta el 0.4\% (en peso de cemento) de cloruros totales, valor habitual en la normativa internacional, salvo el $\mathrm{ACl}$, que fija el límite en el $0.15 \%$ de cloruros solubles en agua.

El objetivo del presente trabajo ha sido estudiar el comportamiento del hormigón armado utilizado en los cajones de dos muelles (en adelante, Muelle A y Muelle B) construidos en la costa Mediterránea con hormigón de categoría $\mathrm{H}-25$, relación agua/cemento igual a 0.50 , contenido de cemento igual a $300 \mathrm{~kg} / \mathrm{m}^{3} \mathrm{y}$ recubrimiento de $40 \mathrm{~mm}$, y situados en ambiente marino sumergido.

Para realizar el estudio, inicialmente se ha analizado la información correspondiente a la fabricación del hormigón y datos del proyecto de los cajones. Tras la extracción de testigos, en laboratorio se ha inspeccionado el aspecto del hormigón y de las armaduras, para a continuación someterlos a una serie de ensayos de caracterización del material: contenido de cloruros, resistencia a compresión, velocidad de propagación de ultrasonidos, penetración de agua, porosidad abierta, absorción de agua por inmersión y absorción de agua por capilaridad, además de la estimación del módulo de elasticidad y de la permeabilidad al agua a partir de los resultados de algunos de los anteriores ensayos.
En los cajones portuarios estudiados, el hormigón se encuentra continuamente sumergido, quedando vista únicamente la viga cantil sobre el mismo, que forma la plataforma del muelle, y que no era objeto del estudio. Dada la dificultad que entraña la extracción de testigos bajo el mar, se planificó la realización de sondeos verticales desde la viga cantil, como se observa en las Figuras 2 y 4 . En las Figuras 1 y 3 se observa en planta y alzado un croquis del punto de extracción del testigo del cajón.

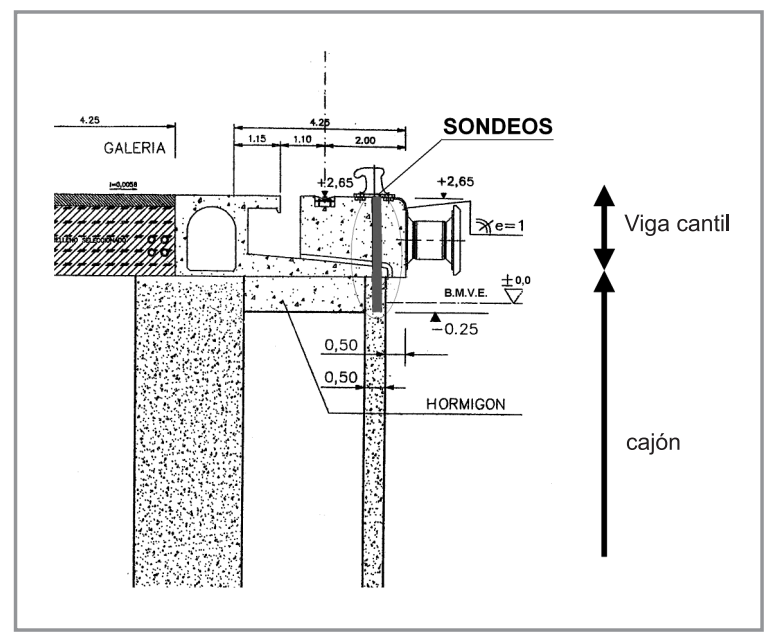

Figura 1. Croquis del punto de extracción del testigo. Alzado

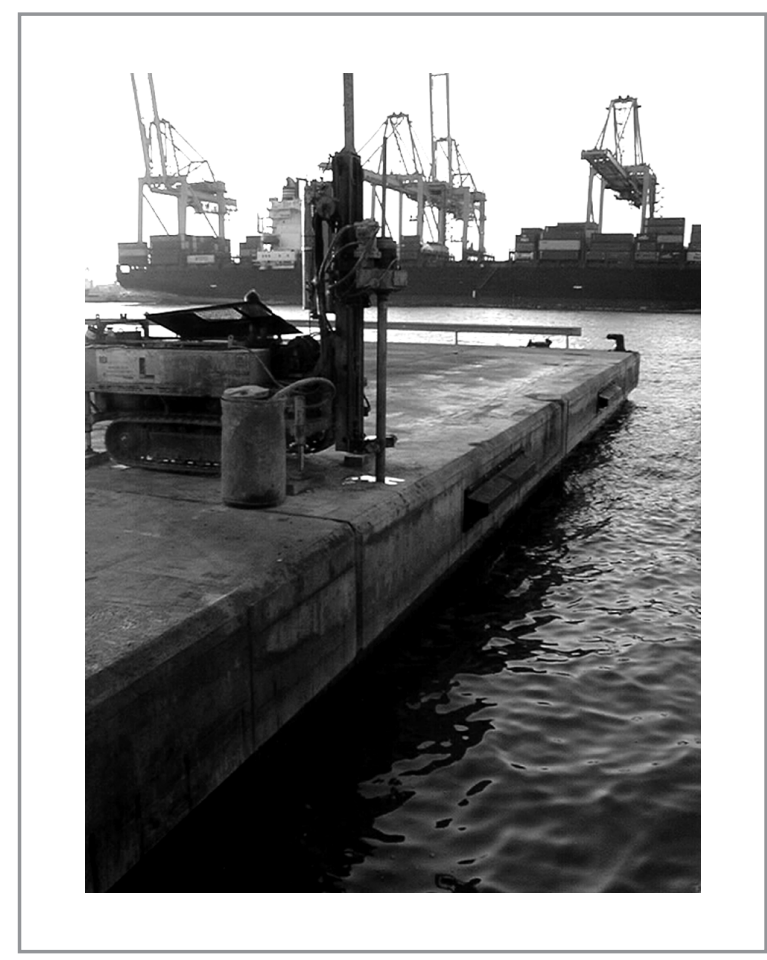

Figura 2. Ejecución del sondeo vertical. Alzado 


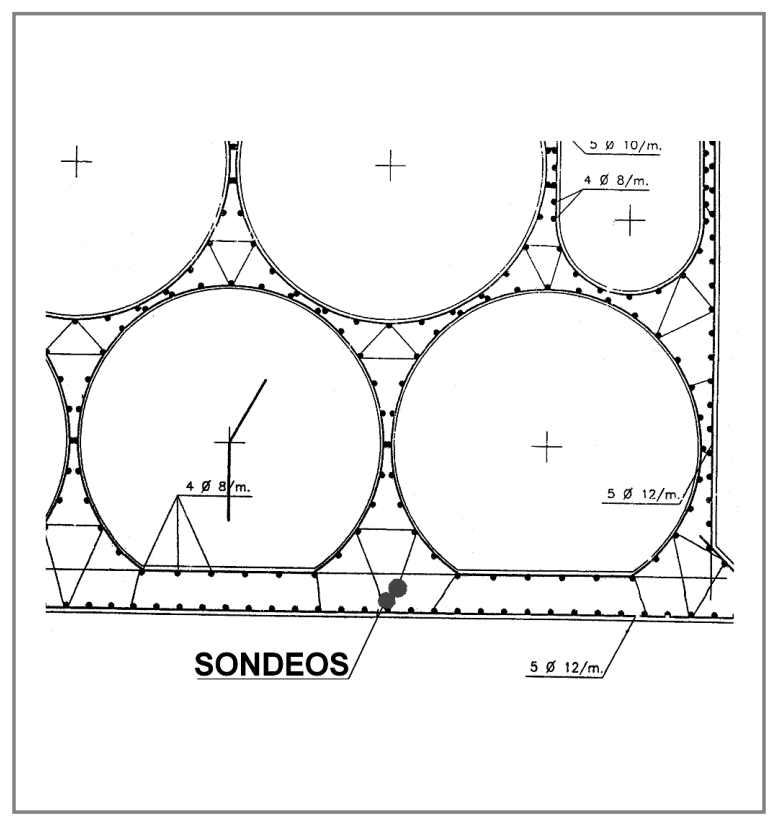

Figura 3. Croquis del punto de extracción del testigo. Planta

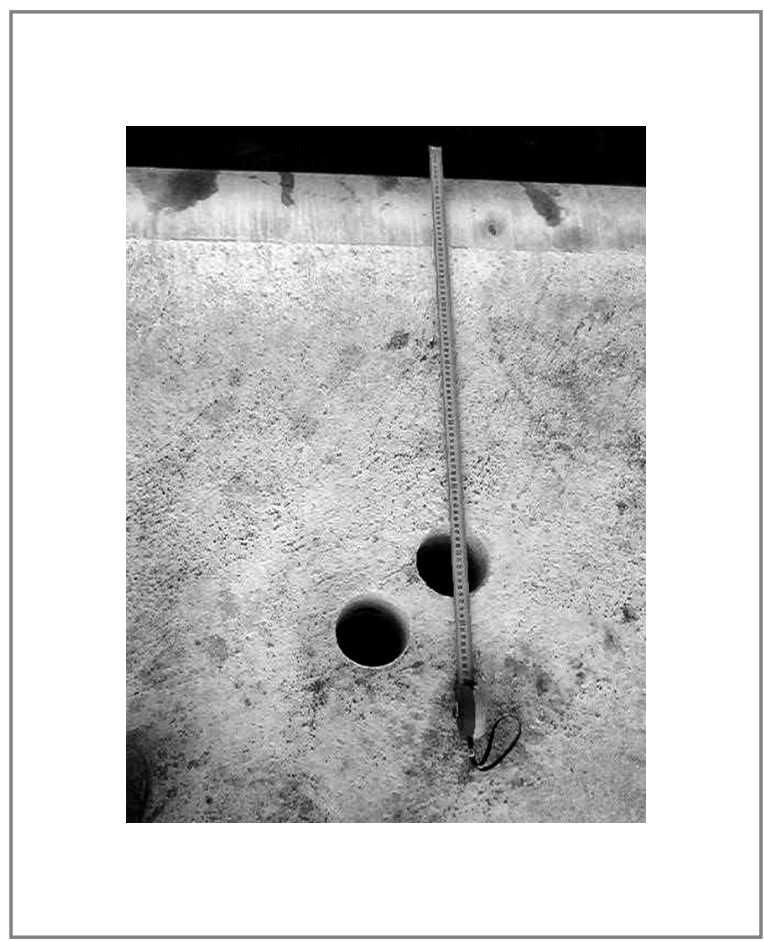

Figura 4. Vista de los sondeos realizados. Planta

En cada uno de los cajones estudiados (tres en cada muelle) se han realizado dos sondeos verticales de $100 \mathrm{~mm}$ de diámetro y $3.00 \mathrm{~m}$ de longitud, salvo en el cajón 2 del Muelle $B$, donde sólo se extrajo un sondeo; por lo tanto, se dispone de seis sondeos del Muelle $A$ (denominados A1 a A6) y cinco del Muelle B (B1 a B5). $\mathrm{D}$ ado que se trataba de determinar el perfil de penetración de cloruros, en cada cajón el primer sondeo se ha intentado situar justo detrás de la armadura más próxima al paramento en contacto con el agua de mar (es decir, el eje del sondeo se sitúa a $11 \mathrm{~cm}$ del paramento), y el segundo sondeo se sitúa a continuación, aunque solapándose $2 \mathrm{~cm}$ con el anterior (es decir, el eje se coloca a $18 \mathrm{~cm}$ del paramento, aproximadamente). Los testigos de hormigón resultantes tienen un diámetro de $83 \mathrm{~mm}$ y una longitud correspondiente al cajón de 145 $\mathrm{cm}$ para el Muelle A y de $50 \mathrm{~cm}$ para el Muelle B.

En los testigos del Muelle $A$ se han cortado tres probetas de $12 \mathrm{~cm}$ de longitud $(7 \mathrm{~cm}$ para el Muelle $B$, salvo una de $11 \mathrm{~cm}$ para el ensayo de resistencia) para realizar los ensayos de resistencia a compresión (U NE 83.304/84) y de medida de la velocidad de transmisión de ultrasonidos (UNE 83-308-93), de porosidad (RILEM CPC 11.3), absorción (BS1881: Part 122) y penetración de agua (UNE 83.309-90), y de ascensión capilar (RILEM CPC 11.2).

A partir de los datos de la profundidad de penetración máxima de agua y de la porosidad abierta del hormigón, se realizó una estimación del coeficiente de permeabilidad, según la ecuación desarrollada por Valenta (Valenta, 1970): k = Px²/2ht, donde "k" es el coeficiente de permeabilidad; " $P$ " es la porosidad; " $x$ " es la profundidad de penetración de agua; "h" es la presión hidráulica; y " $t$ " es el tiempo que está actuando la presión.

El análisis de la penetración de cloruros se suele realizar mediante la determinación del perfil de penetración, que se obtiene analizando químicamente (según la norma ASTM C1152) el contenido de cloruros de muestras de hormigón tomadas a diferentes profundidades.

En los testigos extraídos se ha cortado una rodaja de $10 \mathrm{~cm}$ para realizar los ensayos de determinación de cloruros. En esa rodaja se han realizado 3 cortes Iongitudinales paralelos al paramento en contacto con el mar, para así obtener 4 rodajas de $2 \mathrm{~cm}$ de espesor y $10 \mathrm{~cm}$ de longitud, con las que se han realizado los análisis químicos.

En las Figuras 5 y 6 se observa un croquis de distribución de ensayos en el testigo: 


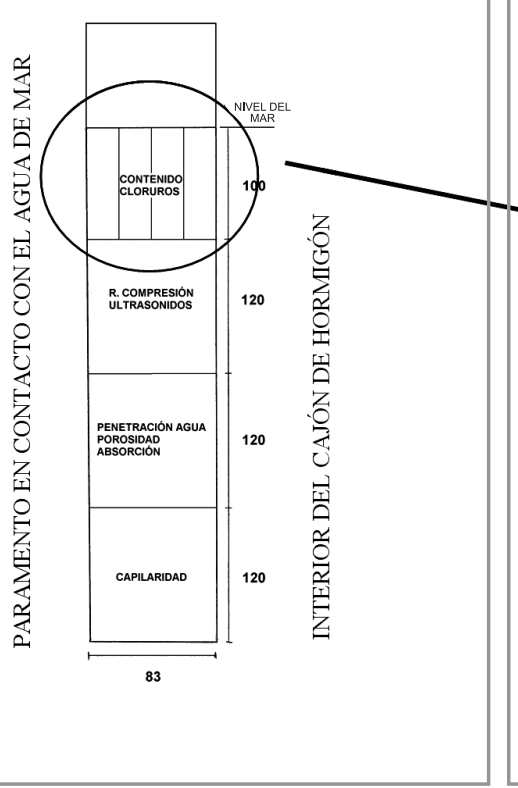

Figura 5. Croquis de división del testigo tipo. Alzado

Figura 6. Detalle de planta del croquis de cortes realizados para obtener el perfil de cloruros.

\section{Resultados}

Las características del hormigón de proyecto fueron (Tabla 1):

Tabla 1. Características del hormigón de proyecto

\begin{tabular}{|c|c|c|c|c|}
\hline & \multicolumn{2}{|c|}{ Muelle A } & \multicolumn{2}{|c|}{ Muelle B } \\
\hline Edad (años) & \multicolumn{2}{|c|}{4.5} & \multicolumn{2}{|c|}{6.5} \\
\hline Hormigón proyecto & \multicolumn{2}{|c|}{$\mathrm{H}-25$} & \multicolumn{2}{|c|}{$\mathrm{H}-25$} \\
\hline Contenido de cemento $\left(\mathrm{kg} / \mathrm{m}^{3}\right)$ & \multicolumn{2}{|c|}{300} & \multicolumn{2}{|c|}{-} \\
\hline Relación agua/cemento & \multicolumn{2}{|c|}{0.50} & \multicolumn{2}{|c|}{-} \\
\hline Recubrimiento proyecto (mm) & \multicolumn{2}{|c|}{40} & \multicolumn{2}{|c|}{40} \\
\hline Tipo de cemento & \multicolumn{2}{|c|}{$\mathrm{I} / 52,5-\mathrm{SR}^{*}$} & \multicolumn{2}{|c|}{-} \\
\hline Tipo de acero & \multicolumn{2}{|c|}{$\mathrm{AEH}-500 \mathrm{~S}$} & \multicolumn{2}{|c|}{ AEH-500 } \\
\hline Armadura horizontal & \multicolumn{2}{|c|}{$5 \Phi 6 / \mathrm{m}$} & \multicolumn{2}{|c|}{$5 \Phi 2 / \mathrm{m}$} \\
\hline Armadura vertical & \multicolumn{2}{|c|}{$7 \Phi 2 / \mathrm{m}$} & \multicolumn{2}{|c|}{$4 \Phi 8 / \mathrm{m}$} \\
\hline \multirow{3}{*}{ Resistencias control de calidad (N/mm²) } & Cajón 1 & 38.4 & Cajón 1 & 29.8 \\
\hline & Cajón 2 & 32.6 & Cajón 2 & 28.7 \\
\hline & Cajón 3 & 37.5 & Cajón 3 & 26.8 \\
\hline \multirow{3}{*}{$\begin{array}{l}\text { Contenido de cloruros }(\%) \text { de los materiales } \\
\text { en control de calidad }\end{array}$} & Cemento & 0.011 & \multirow{3}{*}{\multicolumn{2}{|c|}{ - }} \\
\hline & Agua & 0.034 & & \\
\hline & Áridos & 0.005 & & \\
\hline
\end{tabular}

* $\mathrm{SR}=$ cemento resistente a sulfatos 
De la inspección visual de los testigos hay que destacar que en general no se observaban fisuras, juntas, presencia de cuerpos extraños, porosidad el evada ni ningún otro tipo de anomalía destacable, salvo la presencia de unas coqueras puntuales. El hormigón tenía un color uniforme, y el árido y la pasta estaban homogéneamente distribuidos. El árido era de machaqueo, con un tamaño máximo de $20 \mathrm{~mm}$. En el testigo B5 (correspondiente al Muelle B) se observa una fisura longitudinal y una coquera de gran tamaño.

Los sondeos cortaron en algunos casos armaduras del cajón, pero en ningún caso se observaban signos de corrosión en dichas armaduras (Figuras 7 y 8 ).

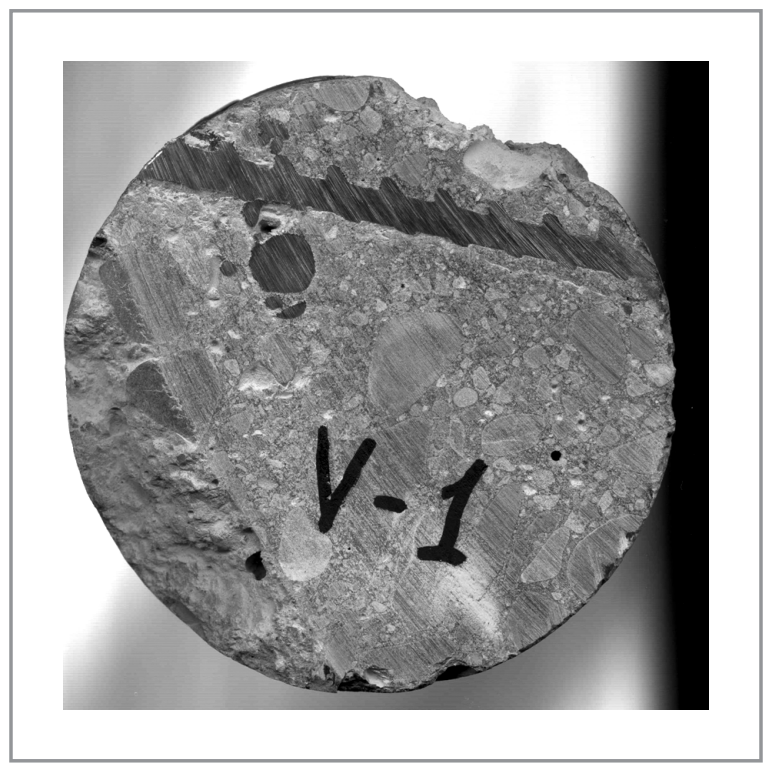

Figura 7. Testigo B1: armadura longitudinal y transversal

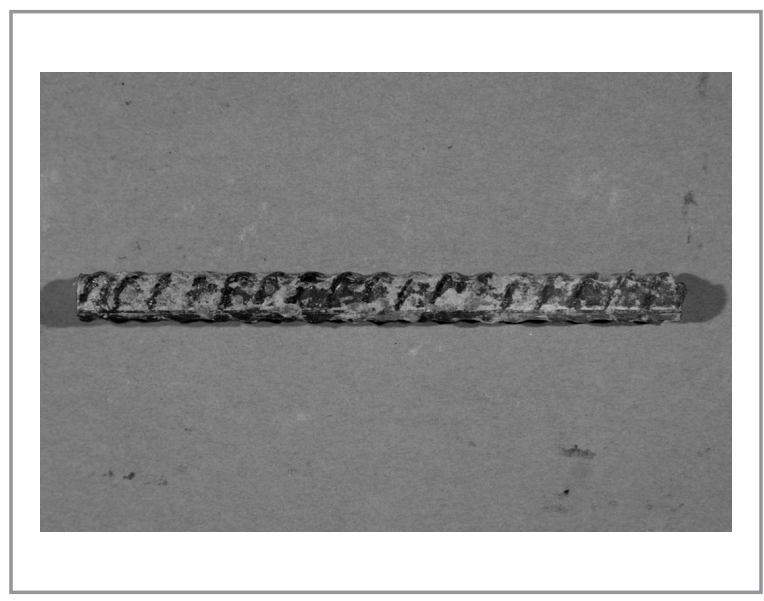

Figura 8. Testigo B1: armadura transversal sin corrosión. Barra extraída de la muestra de hormigón de la foto anterior.
3.1 Resistencia a compresión y velocidad de ultrasonidos

Los resultados obtenidos para los dos Muelles (testigos A y B) se recogen en la Tabla 2; el módulo de elasticidad del hormigón se ha estimado a partir de la velocidad de ultrasonidos (ondas $\mathrm{S}$ ): $\mathrm{E}=2 *(1+\sigma)^{*} \rho * \mathrm{~V}_{\mathrm{S}}{ }^{2}$, donde $\sigma$ es el coeficiente de Poisson, $\rho$ es la densidad y $\mathrm{V}_{\mathrm{s}}$ es la velocidad de ultrasonidos (ondas S).

Tabla 2. Resistencia a compresión, velocidad de ultrasonidos y módulo de elasticidad

\begin{tabular}{||c|c|c|c|c||}
\hline \hline TESTIGO & $\begin{array}{c}\text { ULTRASONIDOS } \\
\text { ONDAS P }(\mathrm{m} / \mathrm{s})\end{array}$ & $\begin{array}{c}\text { R. COMPRESIÓN } \\
\left(\mathrm{N} / \mathrm{mm}^{2}\right)\end{array}$ & $\begin{array}{c}\text { ULTRASONIDOS } \\
\text { ONDAS S }(\mathrm{m} / \mathrm{s})\end{array}$ & $\begin{array}{c}\text { M. ELASTICIDAD } \\
\left(\mathrm{N} / \mathrm{mm}^{2}\right)\end{array}$ \\
\hline A2 & 4.419 & 44.3 & 2.270 & 30.286 \\
\hline A3 & 4.581 & 48.7 & 2.460 & 35.465 \\
\hline A6 & 4.529 & 44.6 & 2.390 & 33.260 \\
\hline B2 & 4.467 & 44.5 & 2.270 & 30.698 \\
\hline B5 & 4.439 & 42.8 & 2.450 & 33.588 \\
\hline \hline
\end{tabular}

\subsection{Penetración de agua y porosidad}

Los resultados del ensayo de penetración de agua y del de porosidad abierta se muestran en la Tabla 3, así como los valores de la permeabilidad al agua del hormigón, estimados a partir de los resultados de los dos ensayos anteriores, según el método desarrollado por Valenta y que anteriormente se ha comentado.

Tabla 3. Profundidad de penetración de agua, porosidad y permeabilidad

\begin{tabular}{||c|c|c|c|c||}
\hline \hline TESTIGO & $\begin{array}{c}\text { PROF. MÁX. } \\
(\mathrm{mm})\end{array}$ & $\begin{array}{c}\text { PROF. MED. } \\
(\mathrm{mm})\end{array}$ & $\begin{array}{c}\text { POROSIDAD } \\
(\%)\end{array}$ & $\begin{array}{c}\mathrm{K}_{\text {est }}\left(\times 10^{-12}\right) \\
(\mathrm{m} / \mathrm{s})\end{array}$ \\
\hline A2 & 44.0 & 31.5 & 18.5 & 6.08 \\
\hline A3 & 45.0 & 28.7 & 19.0 & 6.64 \\
\hline A6 & 35.0 & 22.7 & 17.1 & 3.61 \\
\hline B2 & - & - & 15.7 & - \\
\hline B5 & 51 & $>30$ & 17.8 & 7.99 \\
\hline \hline
\end{tabular}

\subsection{Absorción de agua y capilaridad}

Los resultados del ensayo de absorción de agua por inmersión y del de absorción de agua por capilaridad se muestran en la Tabla 4.

Tabla 4. Absorción de agua por inmersión y absorción de agua por capilaridad

\begin{tabular}{||c|c|c|c|c||}
\hline \multirow{2}{*}{ TESTIGO } & \multirow{2}{*}{$\begin{array}{c}\text { ABSORCIÓN } \\
(\%)\end{array}$} & \multicolumn{2}{|c||}{ CAPILARIDAD } & ALTURAASCENSIÓN \\
\cline { 3 - 5 } & $\left(\mathrm{g} / \mathrm{mm}^{2}\right)\left(\times 10^{-3}\right)$ & $(\mathrm{mm} / \sqrt{\mathrm{min}})$ & 0.13 & 45.0 \\
\hline A2 & 3.76 & 4.6 & 0.6 & 35.1 \\
\hline A3 & 3.98 & 5.5 & 0.16 & 41.4 \\
\hline A6 & 3.33 & 5.7 & 0.17 & 61.9 \\
\hline B2 & 3.37 & 7.2 & 0.16 & $>70$ \\
\hline B5 & 4.34 & 8.8 & 0.20 & \\
\hline
\end{tabular}




\subsection{Profundidad de penetración de cloruros}

Los resultados obtenidos en los ensayos químicos se muestran en los Figuras 9 a 14, en los que se representa la concentración de cloruros totales (expresados en \% en peso de cemento) en función de la profundidad (en cm) a la que se toma la muestra; la línea horizontal representa el contenido de cloruros máximo admisible por la EHE ( $0.4 \%$ en peso de cemento).

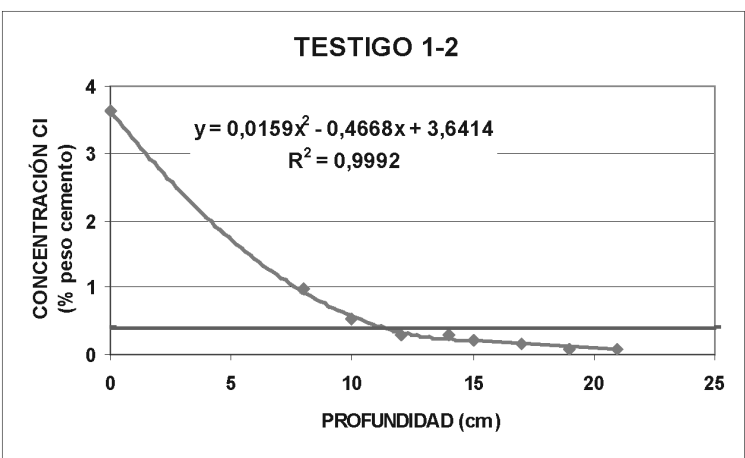

Figura 9. Perfil de penetración de cloruros del Cajón 1 del Muelle A

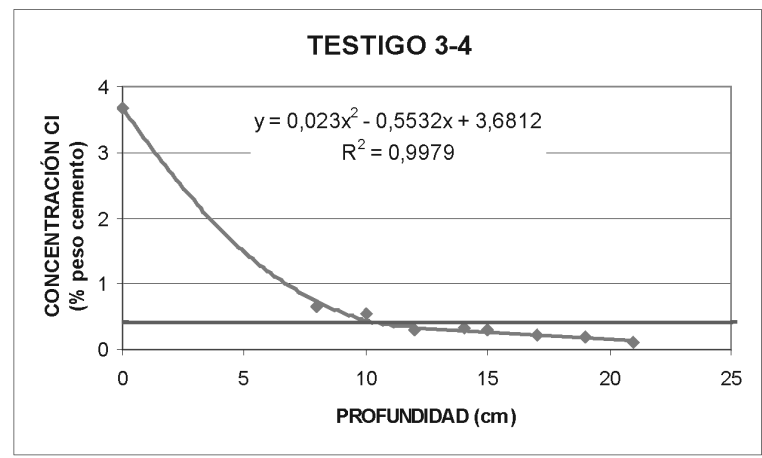

Figura 10. Perfil de penetración de cloruros del Cajón 2 del Muelle A

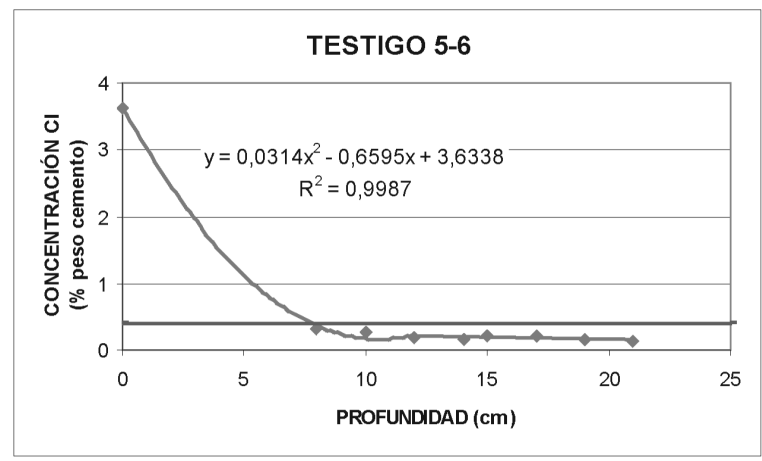

Figura 11. Perfil de penetración de cloruros del Cajón 3 del Muelle A

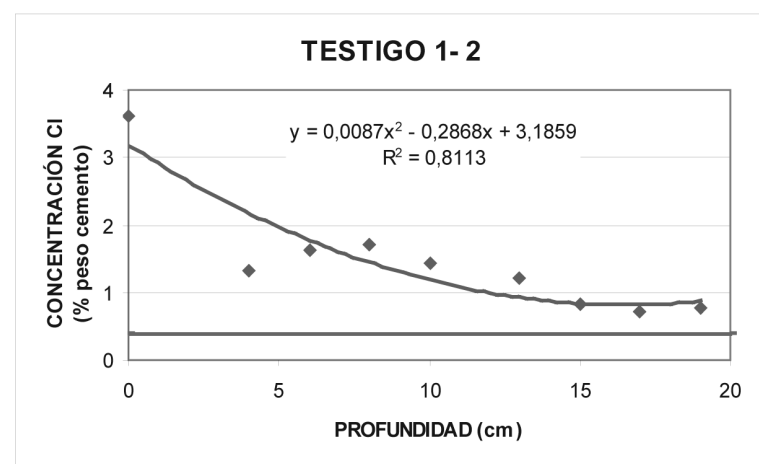

Figura 12. Perfil de penetración de cloruros del Cajón 1 del Muelle B

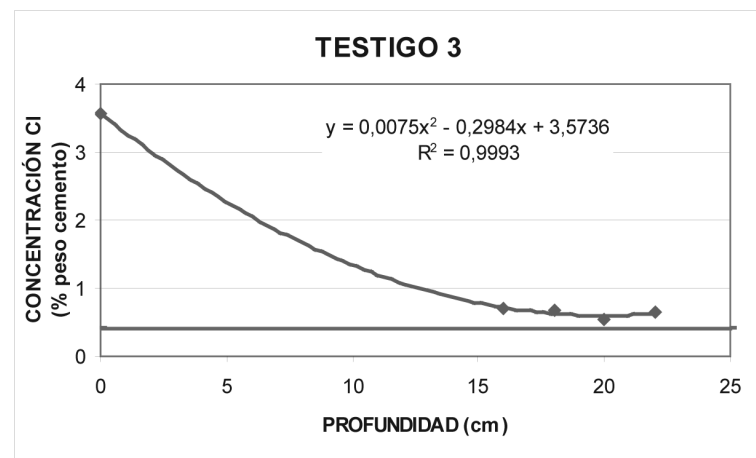

Figura 13. Perfil de penetración de cloruros del Cajón 2 del Muelle B

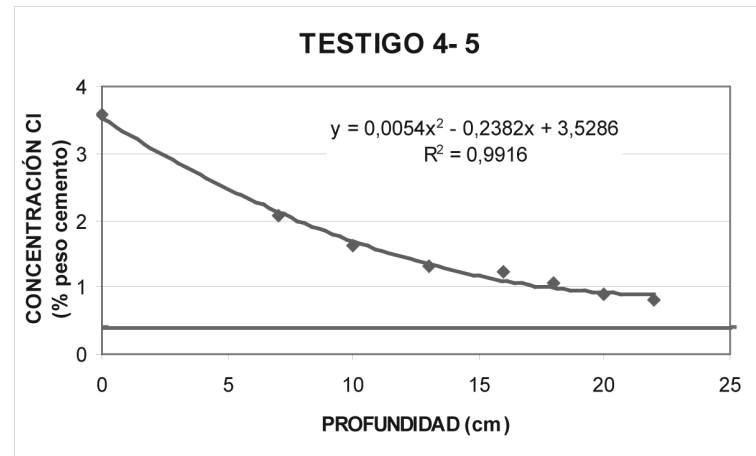

Figura 14. Perfil de penetración de cloruros del Cajón 3 del Muelle B

\section{Discusión}

4.1 Resistencia a compresión, ultrasonidos y módulo de elasticidad

Para el Muelle A, los valores de la velocidad de ultrasonidos permiten calificar la calidad del hormigón 
como excelente. Los valores de resistencia a compresión son muy elevados y uniformes, al igual que los del módulo de elasticidad.

Si se comparan estos valores con los resultados extraídos del control de calidad, se observa un importante incremento de la resistencia del hormigón durante los cuatro años de servicio del muelle: un $16 \%$ para el cajón 1; un 36\% para el cajón 2 y un 30\% para el cajón 3 .

En cuanto al Muelle B, los valores de la velocidad de ultrasonidos permiten calificar la calidad del hormigón como buena, aunque rozando el nivel de excelente. Al igual que en el Muelle A, los valores de resistencia a compresión también son muy elevados y uniformes, como los del módulo de elasticidad.

Hay que destacar el altísimo incremento de resistencia que se observa comparando estos resultados con los extraídos del control de calidad: un 49\% para el cajón 1 y un 60\% para el cajón 3, tras seis años de servicio del muelle.

\subsection{Penetración de agua y porosidad}

La Instrucción EHE, en su artículo 37.3.2., establece que un hormigón se considera suficientemente impermeable al agua si los resultados del ensayo de penetración de agua cumplen simultáneamente que la profundidad máxima sea menor o igual que $50 \mathrm{~mm}$ y la media menor o igual que $30 \mathrm{~mm}$, realizando el ensayo según la norma UNE 83.309-90.

Los resultados de penetración de agua obtenidos en los testigos indican que el hormigón del Muelle $\mathrm{A}$ cumple las condiciones exigidas por la EHE para obtener una adecuada impermeabilidad al agua, salvo en el caso de la profundidad media del testigo A2. En todo caso, los testigos A2 y A3 quedan muy cerca de los límites de validez, mientras que el testigo $A 6$ cumple la prescripción con cierta holgura.

Según el CEB (CEB, 1998), se puede estimar la calidad del hormigón a partir de los resultados de porosidad y de permeabilidad; la porosidad que se ha obtenido en los tres testigos permite calificar el hormigón como de calidad mala, si bien el testigo A6 está en el límite que permitiría clasificarlo como de calidad media. El coeficiente de permeabilidad que se ha calculado a partir de los resultados de penetración de agua y de porosidad en los tres testigos permite calificar el hormigón como de calidad media. El testigo A6 sería algo mejor que los otros dos.
En cuanto al Muelle B, el testigo B6 no cumple las condiciones exigidas por la EHE para obtener una adecuada impermeabilidad al agua. La porosidad que se ha obtenido permite calificar el hormigón del testigo B2 como de calidad media y el del B6 como de calidad mala. El testigo B6 muestra una permeabilidad que calificaría al hormigón como de calidad media.

\subsection{Absorción de agua y capilaridad}

Según el CEB (CEB, 1998), a partir de estos resultados del coeficiente de absorción de agua $\left(A_{b}\right)$, los hormigones de los tres testigos son de calidad media. De los tres testigos, el mejor sería el A6. En función de los resultados del ensayo de capilaridad, el hormigón de los tres testigos sería de calidad media, aunque el testigo A2 sería algo mejor que los otros dos.

Según el ensayo de absorción, el hormigón del testigo B2 es de calidad media y el del B6 es de calidad mala. A partir del ensayo de capilaridad, el hormigón de los dos testigos sería de calidad media, aunque el valor obtenido en el testigo B6 está casi en el límite que permitiría calificarlo como de calidad mala.

\subsection{Profundidad de penetración de cloruros}

La Instrucción EHE (art. 30.1.) limita el contenido total de ión cloro del hormigón, expresado (dada la capacidad de fijación y neutralización de los cloruros que tiene el cemento) en términos de porcentaje en peso del cemento, a un máximo del $0.4 \%$. Por lo tanto, en los dos primeros cajones ensayados del Muelle $A$ hay un contenido excesivo de cloruros hasta una profundidad de unos $11 \mathrm{~cm}$, mientras que en el tercero se situaría el umbral a unos $7 \mathrm{~cm}$ de profundidad, como se observa en las Figuras 9 a 11.

En los tres sondeos del Muelle $B$ hay un contenido excesivo de cloruros hasta una profundidad superior a los $20 \mathrm{~cm}$ (Figuras 12 a 14).

\subsection{Resumen de resultados de calidad del hormigón \\ En función de los diferentes ensayos realizados, la calidad del hormigón de los cajones estudiados se podría clasificar cualitativamente según la Tabla 5. Esta caracterización indica que las propiedades mecánicas del hormigón son muy buenas (resistencias en todos los casos superiores a $40 \mathrm{~N} / \mathrm{mm}^{2}$ y elevadas velocidades de ultrasonidos), no así la valoración global de la durabilidad, que podría calificarse como "Media".}


Tabla 5. Calidad del hormigón según los diferentes ensayos realizados (CEB, 1998; Fernández, 1989)

\begin{tabular}{||c|c|c|c|c|c||}
\hline \multirow{2}{*}{ Ensayo } & \multicolumn{3}{|c|}{ Muelle A } & \multicolumn{2}{c||}{ Muelle B } \\
\cline { 2 - 6 } & Cajón 1 & Cajón 2 & Cajón 3 & Cajón 1 & Cajón 3 \\
\hline Ultrasonidos & Excelente & Excelente & Excelente & Buena - excelente & Buena - excelente \\
\hline Porosidad & Mala & Mala & Media - mala & Media & Mala \\
\hline Permeabilidad & Media & Media & Media & - & Media \\
\hline Absorción & Media & Media & Media & Media & Mala \\
\hline Capilaridad & Media & Media & Media & Media & \\
\hline
\end{tabular}

\section{Conclusiones}

- En estos muelles estudiados, aunque la resistencia del hormigón resulte elevada, se observa que el hormigón contiene una elevada porosidad, que se refleja en unos mediocres resultados de los ensayos de durabilidad.

- Los análisis de concentración de cloruros parecen indicar que para evitar la penetración de grandes cantidades de cloruros es necesario alcanzar calidades del hormigón especialmente buenas. Los hormigones estudiados habían sido curados con agua de mar, pero el efecto de la mayor penetración de cloruros inicial parece difuminarse con el tiempo, según los resultados de otros ensayos que actualmente se están realizando.

- Sin embargo, la inspección visual de las armaduras indica que no hay síntomas de corrosión a pesar de que el contenido de cloruros a esa profundidad sea muy superior al $0.4 \%$ indicado en la $\mathrm{EHE}$, debido a que el acceso de oxígeno hasta las armaduras es escaso al encontrarse el hormigón totalmente saturado de agua. Por lo tanto, en ambiente marino sumergido, el contenido crítico de cloruros que supone el inicio de la corrosión debe ser bastante superior al 0.4\%, y según los datos del Muelle $B$, al menos superior al $1.34 \%$ en peso de cemento.

- De acuerdo con estos resultados, es posible que en ambiente marino sumergido sea suficiente un hormigón tipo $\mathrm{H}-25$, mientras que para los ambientes de carrera de mareas y atmosférico, donde sí hay una exposición mayor al oxígeno atmosférico, se necesite utilizar hormigones de superior calidad.

\section{Bibliografía}

ACI American Concrete Institute (2002), "Fixed O ffshore Concrete Structures. ACI 375R". En ACI Manual of Concrete Practice 2002 Part 5. Design and Construction Practices. D etroit. USA.

BSI British Standards Institution (1984), "British Standard

Code of Practice for M aritime Structures. Part I. General Criteria. BS 6349". London.

CEB Comité Euro-International du Béton (1998), "Strategies for testing and assessment of concrete structures. Guidance Report". Boletín CEB no 243. Lausanne.

Fernández Cánovas M. (1989), “Hormigón”, 364-400. Madrid: E.T.S. Ingenieros de Caminos.

FIP Fédération Internationale de la Précontrainte (1985),

"Design and construction of concrete sea structures. FIP Recommendations". London.

Mehta P. K. y M onteiro P. J. M. (1986), Concrete. Structure, Properties and Materials (113-178). Ed. Prentice Hall. M inisterio de Fomento (1998), Instrucción de Hormigón Estructural: EHE. Madrid: Ed. Centro de Publicaciones. RILEM (1985), Technical Committee 32-RCA. Subcommittee "Long-time Studies" , "Seawater attack on concrete and precautionary measures". Materials and Structures, Vol. 18, No 105, 223-226. Valenta, 0. (1970), "The permeability and durability of concrete in aggressive conditions". Proceedings of Tenth International Congress on Large Dams. M ontreal. 